\title{
Long Term Induction of Kindled Seizures in Rats: Interhemispheric Factors
}

\author{
JOSÉ N. NOBREGA AND JOHN GAITO
}

SUMMARY: Previous research indicated that sequential alternation of stimulation of certain homologous brain areas via chronically implanted electrodes resulted in oscillation of high and low latencies for convulsions. This phenomenon suggested the establishment of interhemispheric facilitatoryinhibitory effects as a result of repeated stimulation of the two brain sites. In the present study, the latency oscillation pattern was observed in split-brain rats as well as in bilaterally stimulated controls, but not in rats stimulated on one side only. Significant differences were observed between split-brain and control rats in terms of initial kindling rates, duration of comvalsions and type of oscillation. Results are discussed in the context of possible interhemispheric mechanisms involved in long term kindling.

RÉSUMÉ: Des recherches antérieares araient indiqué que des stimulations alternées de façon séquentielle de certaines régions cérébrales homologues par la voie d'électrodes implantées chroniquement résultaient en des oscillations convalsives du haute ou de basse latence. Ce phénomène semble indiquer qu'il s'installe des effets facilitaterarsinhibitears interhémisphériques à la suite de stimulations répétées des deax sites cérébraux. All cours des expériences présentes le tableau d'oscillation dans la latence s'est maintenu chez des rats avec section interhémisphérique ainsi que rhez des contrôles stimulés bilatéralement. mais non rhez les rats stimulés sealement unilatéralement. Il existe des différences significatives entre les rats avec section interhémisphérique er les rats contrôles en ce qui concerne le taux intial de kindling, la durée des convalsions et le type d'oscillation. Nous discutons ces résultats en rapport avec le contexte de mécanismes interhémisphériques impliqués dans le kindling à long terme.

From the Department of Psychology, York University.

Reprint requests to: José N. Nobrega, 4700 Keele St., Downsview, Ontario, Canada M3J IP3.
Repeated low-level electrical stimulation of any of a variety of brain regions induces progressive changes which culminate in clonic convulsions. As stimulation trials proceed, the animal's behavior changes from an initial stage of increased exploratory behavior (Stage 1) to behavioral automatisms usually involving facial contractions, chewing movements, eye closure and salivation (Stage 2), and finally to full-blown motor seizures (Stage 3). This sequence of events has been called the "kindling effect" (Goddard et al., 1969).

Once the motor seizure stage has been achieved, usually after 10-15 trials with rats, subsequent stimulation trials will induce the clonic convulsions (CC), even if weeks or months elapse between stimulations. Thus, a permanent brain change is thought to result from kindling (Goddard et al., 1969). Kindled seizures characteristically have a latency measurable in seconds between the onset of stimulation and the onset of the clonus. They last for several seconds after the electrical stimulation is terminated. The effect is remarkably resistant to a number of experimental manipulations (Gaito, 1974). Careful morphological analyses have indicated that the effect is not due to edema, ion deposition, or any form of damage to the stimulated tissue (Goddard et al, 1969; Goddard and Douglas, 1975). Although the amygdaloid complex appears to be particularly sensitive to kindling, the effect may be obtained from a number of different brain regions, especially those within the limbic system (Goddard et al., 1969; Cullen and Goddard, 1975).
Both transfer and interference effects are obtained when two homologous or non-homologous brain sites are successively kindled. After kindled seizures have been obtained at one brain site (primary site), fewer stimulation trials are usually necessary to achieve the CC stage on the contralateral site (secondary site), indicating the occurrence of facilitation or positive transfer from primary to secondary site. However, latencies for the secondary site are reported to be higher than those for the primary site (Goddard et al., 1969; Racine, 1972; McIntyre and Goddard, 1973), indicating negative transfer. If stimulation is then reapplied at the primary site, a temporary suppression of convulsions is observed and it takes a few trials (although less than originally) to evoke CC's from the primary site. This event suggests the occurrence of interference or negative transfer from secondary to primary site (McIntyre and Goddard, 1973).

We became interested in the time course of these transfer/interference phenomena, i.e., in the question of what would happen if stimulation trials were extended to more than one primary-secondary-primary cycle. Our initial expectation was that after a few alternations of stimulation between primary and secondary sites, the positve and negative transfer effects would neutralize each other and CC's obtained from two homologous sites would be indistinguishable, both in terms of number of trials necessary to elicit a given number of convulsions and in terms of latencies to convulsion. The basic paradigm in our experiments consisted of inducing a given number of CC's (usually 6) from 
stimulation of one brain site, then stimulating the contralateral site until the same criterion was achieved. Stimulation was then reapplied at the primary site, then at the secondary, and so on in a schedule of sequential alternation of unilateral stimulation until 10 phases (each with 6 CC's) were completed. Using this paradigm, we found that the number of trials to criterion usually became stable after a few phases of alternations, but latencies between adjacent phases did not stabilize. Instead, an oscillation pattern became established. Stimulation of one site resulted in consistently low latencies and stimulation of the contralateral site resulted in consistently high latencies. We have referred to this occurrence as the "oscillation effect" (Gaito, 1976a). To illustrate the phenomenon, Figure 1 shows the latency functions obtained in our experiments of sequential alternation of unilateral stimulation.

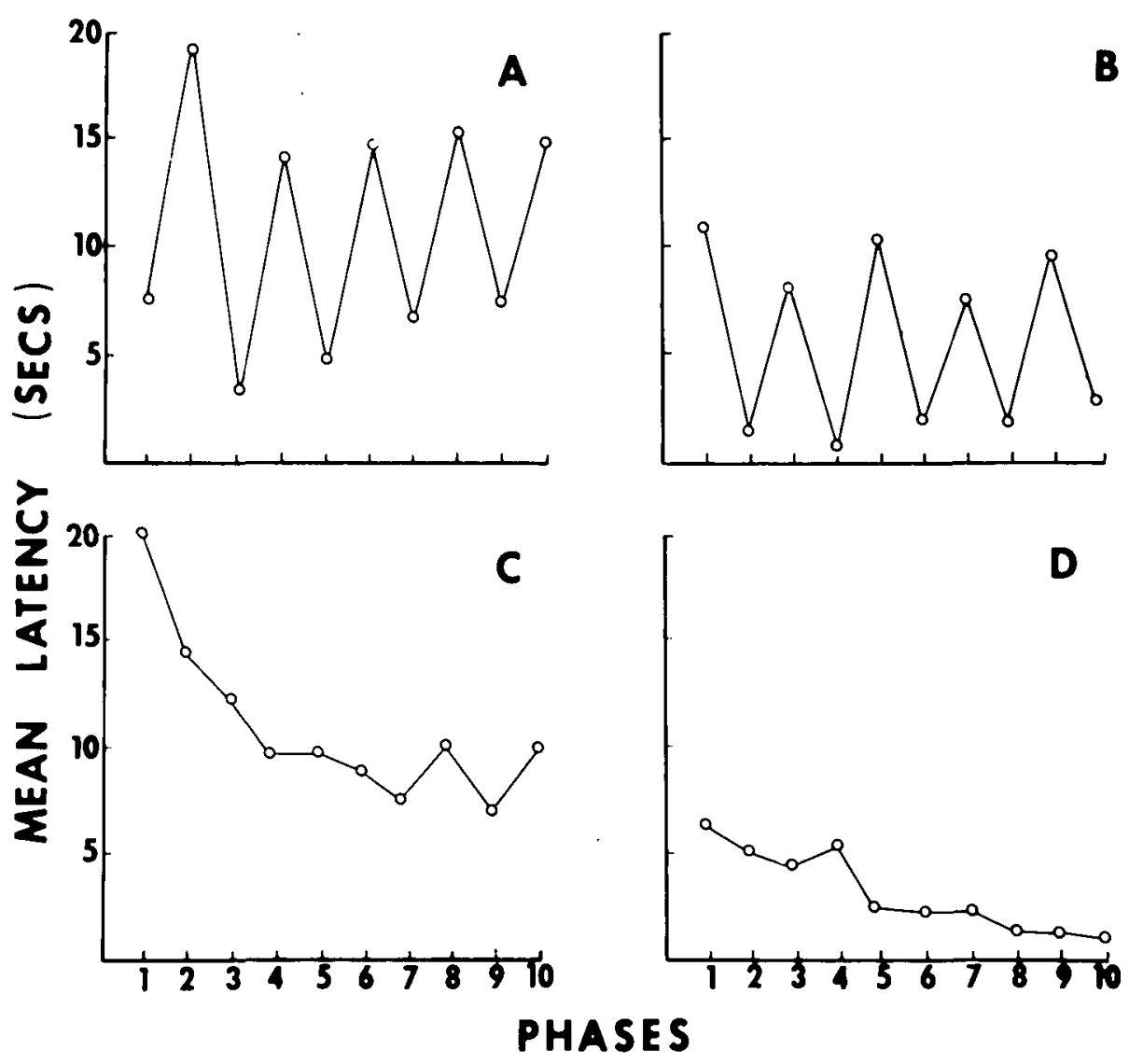

Figure 1 -Examples of latency functions for 4 animals submitted to 10 phases of sequential alternation of stimulation. Odd-numbered phases correspond to primary site stimulation, even-numbered phases to secondary site stimulation.
The top graphs in the figure are examples of the two basic types of oscillation. In Figure 1A, latency values for the primary site (phases 1 , $3,5,7$, and 9) are consistently lower than those for the secondary site; this pattern is primary oscillation (PO). Figure 1B illustrates the opposite pattern (secondary oscillation SO), which occurs nearly as often as primary oscillation. Figure $1 \mathrm{C}$ shows an example of non-oscillation (NO), which occurs in approximately $25 \%$ of the animals in latency data, but more frequently in criterion and duration measures. Figure ID shows the latency function of a typical control animal submitted to 10 blocks of 6 trials, with all convulsions elicited from the same brain site.

Similar to kindling, oscillation appears to be an extremely reliable phenomenon, occurring in approximately $75 \%$ of all rats tested. This is significantly greater than that expected on the basis of chance alone ( $p$ of oscillation is .17). ${ }^{1}$ Systematic

\section{.} us to the view that the oscillation effect was not an artifact, but possibly resulted from the establishment of excitatory-inhibitory patterns between the two hemispheres, as a result of repeated stimulation. If this was the case, it was conceivable that such patterns might be disrupted by radical transection of interhemispheric connections. The present study was designed to test the effects of sectioning interhemispheric connections on the oscillation effect.

Recent kindling studies, using split-brain animals, have reported no differences in the kindling rates of split-brain and control rats (McIntyre, 1975; McCaughran et al., 1976). Much faster initial kindling rates have been reported in splitbrain cats (Wada and Sato, 1975). However, since in each of these experiments there were differences

\footnotetext{
1 The hypothesis that the oscillation patterns are random ones can be assessed by the one sample runs test (Hoel. 1954). Using the requirement of 8 or 10 phases of oscillation out of 10 phases would provide 8, 9, or 10 runs. The $p$ of 8.9 . and 10 runs is 1270 , .0317 , and .0079 , respectively: the $p$ of 8,9 , or 10 runs is the sum of these $p^{\circ} s^{\circ} .1666$ or .17 . Thus the $p$ that these apparent systematic patterns are random ones would be 17 .
} 
among animals in terms of extent of lesions, it was desirable to reassess this aspect as well as overall facilitation/interference phenomena in split-brain rats.

\section{METHODS}

Experimental Animals

Fifty-six male Wistar rats weighting from $300 \mathrm{gm}$ to $400 \mathrm{gm}$ were divided into 4 groups according to surgical treatment received and stimulation schedule. The animals were housed in individual metal cages with water and food available at all times. A 12-hr dark-light period was maintained throughout the experiment.

\section{Surgery and Electrode Implantation}

Seventeen animals were submitted to split-brain surgery. Under sodium pentobarbital anesthesia, each animal was positioned in a stereotaxic apparatus and a rectangular bone flap $(4 \mathrm{~mm}$ anterior to bregma to $6 \mathrm{~mm}$ posterior and $2 \mathrm{~mm}$ lateral to the midline) was removed from the skull. The dura was deflected and a sharpened blade was lowered between the hemispheres for the length of the skull opening and then withdrawn. The bone flap was replaced and the skin sutured. Surgery aimed at complete transection of the corpus callosum and all midline structures.

A second group of 16 animals was anesthetized and placed in the stereotaxic apparatus. A bone flap was removed from the skull and the dura was deflected, but no incision was made. A third group of 23 animals, later to be divided into two control groups, was anesthetized and allowed to rest until the time of electrode implantation.

Approximately 6 weeks later, all animals were bilaterally implanted. Electrodes consisted of $.127 \mathrm{~mm}$ nichrome wires twisted together and coated once in epoxylite, with approximately $.8 \mathrm{~mm}$ between the exposed tips. In all cases the electrodes were aimed at the amygdaloid complex, using the coordinates of previous experiments $(.5 \mathrm{~mm}$ posterior to bregma, $4.5 \mathrm{~mm}$ from the midline, $8.5 \mathrm{~mm}$ from skull).

\section{Experimental Procedures}

Stimulation trials began a week after electrode implantation. Each trial consisted of passing a $60 \mathrm{~Hz}$ sine wave current for 30 seconds, using a Lafayette stimulator. The initial intensity was $100 \mu \mathrm{A}$ for all animals; the current was gradually increased, if there was no apparent response after 10 trials, until the optimum intensity was achieved for each animal. In most cases the CC stage was achieved with $100 \mu \mathrm{A}$ and in no case was the intensity level raised beyond $400 \mu \mathrm{A}$.

The split-brain, sham-operated, and 10 unoperated control animals were submitted to 10 phases of sequential alternation, following our standard procedure. Three stimulation trials, at least 1 hour apart, were given each day until $6 \mathrm{CC}$ 's were obtained on the stimulated site. The day after the 6 th $\mathrm{CC}$, stimulation began at the contralateral site and was maintained until the $6 \mathrm{CC}$ criterion was achieved. This alternating procedure was repeated until 10 phases were completed. The remaining 13 rats were assigned to a unilateral control group. For animals in this group, one of the two electrodes was randomly selected and 3 trials were given each day until 10 phases of 6 CC's were obtained from stimulation of that site alone.

\section{Histology}

At the end of the experiment the animals were sacrificed with an overdose of sodium pentobarbital and perfused through the heart with saline and formalin. The brains were removed and kept in a $10 \%$ formalin solution for at least one month. The brains were then frozen and $50 \mathrm{mic}$ ron sections were cut and mounted on microscopic slides which were placed in a photographic enlarger and used as negatives to provide permanent prints of the unstained brain sections. In some cases the sections were stained with cresyl violet. For split-brain rats, sections were made at every $.2 \mathrm{~mm}$ to determine the longitudinal extent of the lesion.

\section{RESULTS}

Examination of the histological plates revealed that failure to place the electrodes in the amygdaloid complex were equally distributed among all groups. Since our previous results indicated no relationship between electrode placement and the oscillation effect, no data were

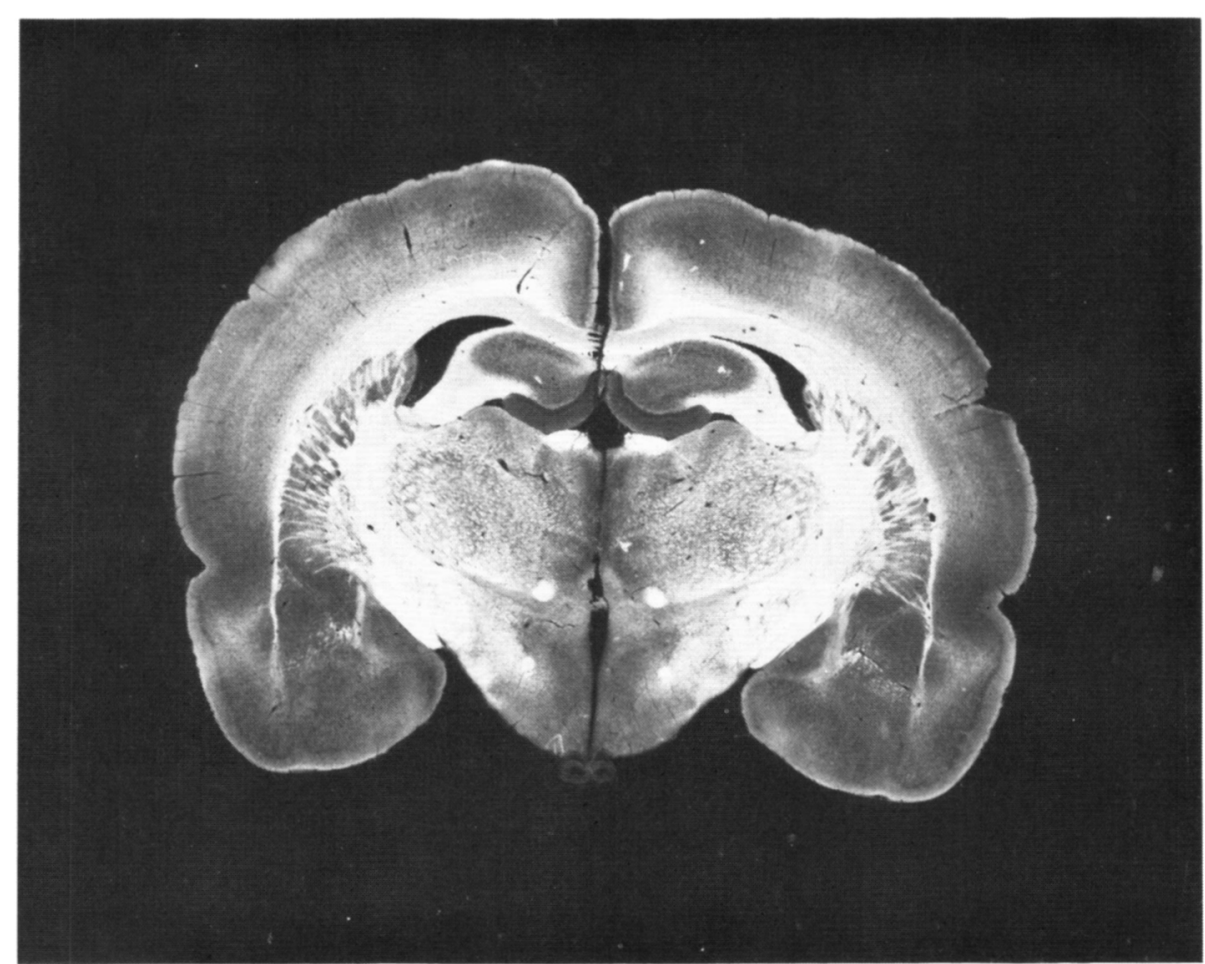

Figure 2-Unstained brain section of a typical split brain animal. 
discarded on the basis of electrode placement. Of the 34 rats used for the analysis, 21 had both electrodes in the amygdala, 7 rats had one electrode in the amygdala and the other in the ventral boundary of the caudate-putamen and globus pallidus. Five rats had both electrodes in the ventral caudate-putamen and 1 rat had one electrode in the globus pallidus and the other in the ventral aspect of the internal capsule.

The histological analysis indicated that the corpus callosum was sectioned in its entirety, as well as subcallosal medial structures in all operated animals (Fig. 2). However, in 3 split-brain animals, the anterior commissure was spared; these animals were included in a sub-group for analysis. Therefore, five groups were distinguished: 1) split-brain including the anterior commissure $(\mathrm{SB}+\mathrm{AC}) ; 2)$ split-brain with the anterior commissure spared (SB $\mathrm{AC})$; 3) unoperated controls (UOC); 4) sham-operated controls (SC); and 5) unilateral stimulation controls (UC).

Kindling was achieved in all animals except for two unoperated control rats which kindled normally on one side, but failed to show CC's on the contralateral side after 100 trials. These animals were discarded. Histological verification revealed that in one of these rats the ineffective electrode was in the stria terminalis/internal capsule region. In the other animal the electrode was in the boundary of the medial forebrain bundle and the internal capsule.

All split-brain rats achieved the CC stage normally. However, certain peculiarities were apparent in this group. In the pre-convulsion behavioral automatism stage, certain responses, such as eye closure, werer restricted to the ipsilateral side. The clonus was clearly limited to the contralateral side of the body. Normal rats in Stage 3 typically rear up on the hind limbs and tail and display the clonus on the two forelimbs. Split-brain animals tended to keep the two ipsilateral limbs on the floor of the test cage and display the clonus on the two contralateral limbs, as previously reported by McIntyre (1975).

TABLE 1

Frequency of occurrence of latency oscillation patterns in the 5 groups

\begin{tabular}{lccccc} 
& $\begin{array}{c}\text { Split-Brain } \\
+ \text { AC }\end{array}$ & $\begin{array}{c}\text { Split-Brain } \\
- \text { AC }\end{array}$ & $\begin{array}{c}\text { Unoperated } \\
\text { Controls }\end{array}$ & $\begin{array}{c}\text { Sham-Operated } \\
\text { Controis }\end{array}$ & $\begin{array}{c}\text { Unilateral } \\
\text { Controls }\end{array}$ \\
\hline $\begin{array}{l}\text { Primary } \\
\text { Oscillation }\end{array}$ & 5 & 3 & 3 & 4 & 0 \\
$\begin{array}{l}\text { Secondary } \\
\text { Oscillation }\end{array}$ & 0 & 0 & 3 & 3 & 2 \\
$\begin{array}{l}\text { Non-Oscillation } \\
\quad \text { N }\end{array}$ & 0 & $\frac{0}{3}$ & $\frac{0}{6}$ & $\frac{1}{8}$ & $\frac{10}{12}$
\end{tabular}

Because of deaths, loosening of electrodes before completion of 10 phases, and occasional instances of tissue degeneration, revealed in the subsequent histological verification, only 34 of the original 56 animals were considered in the analysis.

Several response measures were obtained for all animals. Of primary interest was the occurrence of oscillation in the various groups. This was assessed by plotting mean latencies over ten phases for each animal and noting the direction of the observed differences between adjacent phases. The oscillation effect, defined as consistent positive or negative differences between adjacent pairs of phases, was observed in all groups. Animals in each group were classified as primary oscillators (at least 4 negative differences in the 5 pairs of phases, 1-2, 3-4, 5-6, $7-8,9-10$ ), secondary oscillators (at least 4 positive differences in the 5 pairs), or non-oscillators. Table 1 shows the frequency of occurrence of these behaviors in the five groups for latency data. Of the 22 bilaterally stimulated rats, 21 showed oscillation patterns. This is significantly different from the number expected by chance $(.17 \times 22=3.7)$, using the binomial distribution $(\mathrm{p}<.05)$. In contrast, only 2 of the 12 rats in the unilateral group showed oscillation; this number is not significantly different from that expected by chance $(2.04 ; p>.05)$.

In the 4 bilateral groups, 8 out of 15 animals showing primary oscillation in latency data had electrodes on homologous brain sites; 3 out of 6 animals showing secondary oscillation had electrodes on homologous sites. The one animal showing non- oscillation had equal electrode placements on both sides. Although it may have been a chance occurrence, it is interesting to note that all split-brain rats showed the primary oscillation pattern.

Apart from the classification of each animal in one of the three oscillation categories, the percentage of primary and secondary oscillation shown by each animal in the 10 phases was analyzed. Thus, if for a given animal primary site latencies were lower than the adjacent secondary site latencies for all 10 phases, that animal would have a score of 1.0 on percent of primary oscillation and 0.0 on percent of secondary oscillation. The other response variables analyzed were the following: 3) number of trials to complete phase 1; 4) number of trials to complete phase $2 ; 5)$ mean duration of convulsions in phase 1 ; 6) mean duration of convulsions in phase $2 ; 7$ ) mean duration for the remaining primary phases; 8) mean duration for the remaining secondary phases; 9) mean latency in phase $1 ; 10$ ) mean latency in phase 2 ; 11) mean latency for the remaining primary phases; and 12) mean latency for the remaining secondary phases.

Analyses of variance were performed on each of the 12 dependent variables (each $F$ test for Between Groups sources with 4 and $29 \mathrm{df}$ ), using an IBM 370 computer at York University. Table 2 shows the means and standard deviations for the 5 groups on the 12 dependent measures. Significant differences $(p<.05)$ among the groups were obtained on the following measures: percentage of primary oscillation, number of trials to complete phases 
Table 2

Means (M) and Standard Deviations (SD)

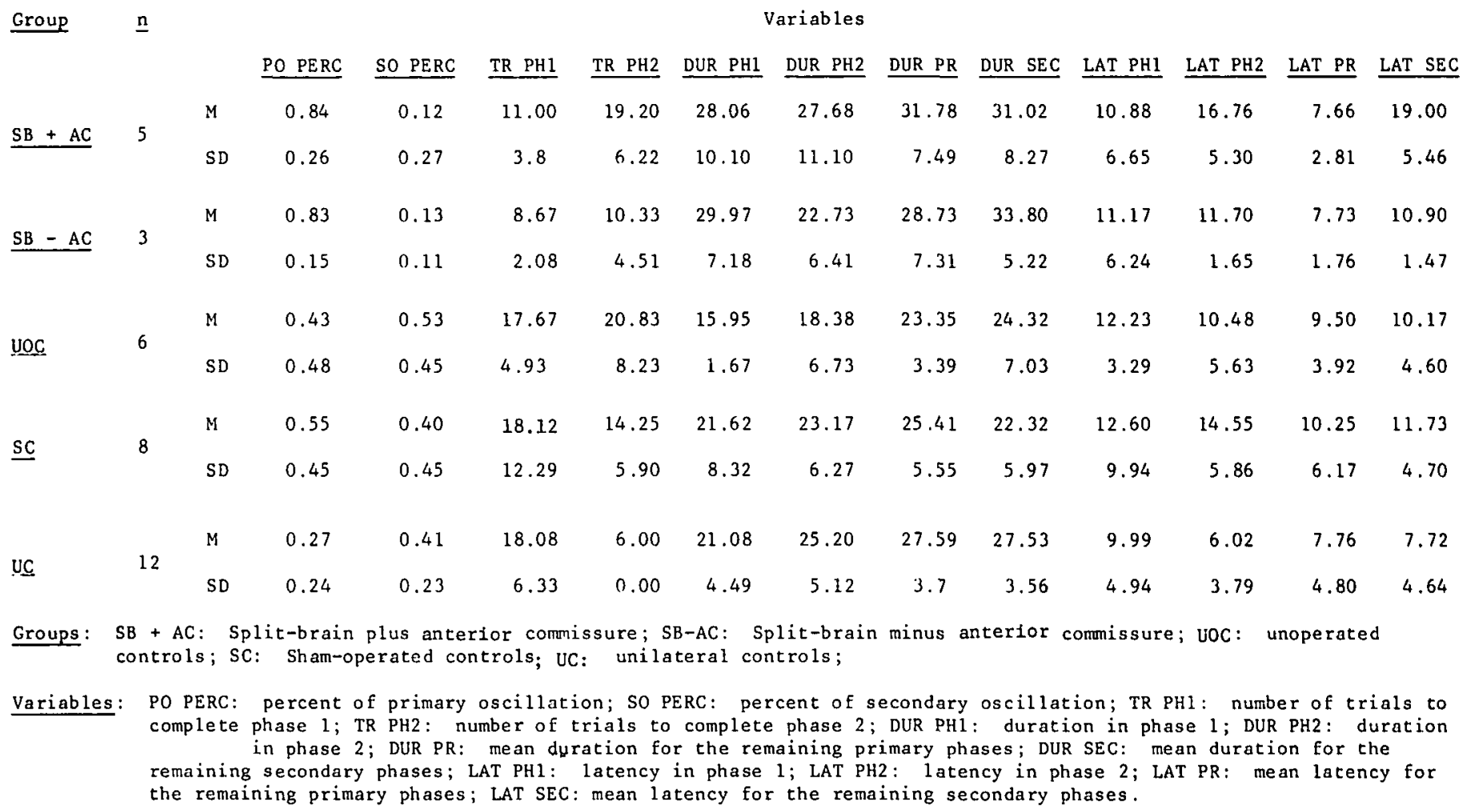

1 and 2, mean duration of convulsions on the primary site, mean latencies in phase 2 , and mean latencies on the secondary site.

Four orthogonal comparisons were performed, each $\mathrm{F}$ test with 1 and $29 \mathrm{df}$. The results were: 1) The means for the unilateral group were significantly lower than those for the bilateral groups in percentage of primary oscillation, number of trials to complete phase 2, and mean latencies in phase 2. 2) The comparison between the means of the two split brain groups and those of the two bilateral control groups indicated that the split-brain groups had a significantly higher percentage of primary oscillation, required fewer trials to complete phase 1 , and had a higher mean duration of convulsions in phase 1 and in the remaining primary and secondary phases than the bilateral control groups. 3) Split-brain + AC animals differed from split-brain - $A C$ animals only in number of trials to complete phase 2 , the mean for the former group being higher than that for the latter.
4) A significant difference on this latter measure was also obtained in the comparison between operated controls and sham-operated animals; the former group had the greater mean.

\section{DISCUSSION}

These results are in agreement with our previous findings that electrode placement does not in itself predict the occurrence or type of oscillation. Primary or secondary oscillation appear to occur whether both electrodes are in the amygdala, one electrode is in the amygdala and the other in a nearby structure, or if both electrodes are in a nearby structure. For example, in two of the secondary oscillators in the present experiment the primary site electrode was in the anterior amygdala and the secondary electrode was in the adjacent caudate area, yet secondary latencies were lower than primary latencies in these animals.

In general, the fact that secondary oscillation occurs nearly as often as primary oscillation (Gaito, Gaito, and Nobrega, 1977) indicates that previous examples of primary oscillation in three phases (McIntyre, 1975; McIntyre and Goddard, 1973) cannot be taken as an absolute finding, but probably reflect the fact that the primary oscillation pattern tends to occur more often than the secondary oscillation pattern.

The finding that split-brain surgery did not disrupt the oscillation effect raises the possibility that the oscillation phenomenon is unilateral in nature, e.i., that it results from intrinsic differences between the two hemispheres instead of from interhemispheric facilitation/interference effects. However, two pieces of evidence argue strongly against this hypothesis. The first relates to observations of reversals in oscillation patterns along the ten phases and to frequent occurrences of what we might call "early" and "late" oscillation in rats which otherwise do not meet our criteria for primary or secondary oscillation over 10 phases. Figure $3 \mathrm{~A}$ illustrates a case of reversal in oscillation after phase 4 . Such occurrences are inconsistent with 


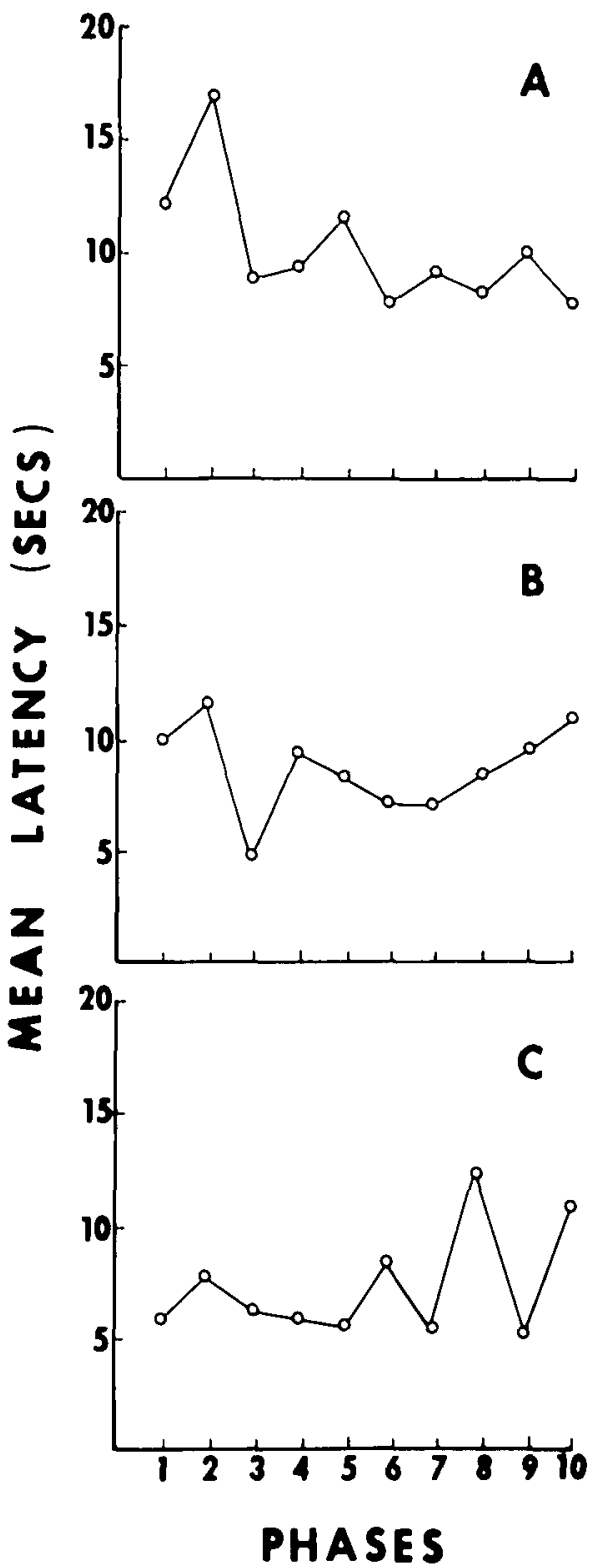

Figure 3-A: "Reversal" in oscillation pattern after phase 5; B: "early oscillation" pattern; C: "late oscillation" pattern.

the hypothesis that each hemisphere simply reacts according to a fixed, innate potential. Figure $3 \mathrm{~B}$ and $3 \mathrm{C}$. show examples of early and late oscillation. In the first case the animal shows an initial oscillation pattern which disappears in subsequent phases. In late oscillation (Figure $3 C$ ), an animal which did not initially display an oscillation pattern begins to do so in the late phases of the experiment. In our long-term experiments where the number of phases has been extended beyond 10 , these three types of changes in latency responses tend to occur more often.

Another argument against the unilaterality of the phenomenon is the fact that in these long-term bilateral stimulation experiments, mean latencies for each of the two sites tend to remain constant after the first few phases. If only one side is stimulated over long periods, latencies tend to decrease faster and approach zero values (Tress and Herberg, 1972); this is often seen in our unilateral stimulation control groups (Figure ID).

The inability to disrupt the oscillation effect by split-brain surgery is a strong indication that at least part of the transfer/interference effects are mediated through the brainstem, as previously suggested by McIntyre (1975). Nevertheless, the uncovered differences between split-brain and control animals suggest that this surgical manipulation does affect longterm kindling in significant ways. In contrast to previous reports splitbrain rats in the present experiment did show faster initial kindling rates in comparison with control animals. Examination of the records of our split-brain rats which lost electrodes before completing the 10 phases and were not included in the analysis confirmed this trend toward faster kindling. The discrepancy between the present data and previous reports may be due to specific differences in stimulation parameters used in each case. In our experiments, we give three 30 -second stimulation trials per day, while the other studies employed one daily trial of 1 second (McCaughran et al., 1976) or 5 seconds (McIntyre, 1975). Still, it is to be noted that in McIntyre's data the total forebrain lesion group required fewer trials to kindle than all other groups, although this difference was not reported to be statistically significant. Differences between the present results and those reported by McCaughran et al. are likely related to different criteria used for assessing the clonic convulsions themselves. While we consider only three stages in the kindling process, those investigators used more stringent criteria which, in addition to the occurrence of clonus (our
Stage 3), required that seizures cause loss of balance in the animals (Racine, 1972).

Two other differences between split-brain and control animals in the present experiment deserve comment. The significant trend in the split-brain group toward primary oscillation may simply reflect the aforementioned fact that in general primary oscillation tends to occur more often than secondary oscillation. Alternatively, it may reflect a trend toward suppression of secondary oscillation in split-brain animals. As to duration of convulsions, the results indicated that split-brain rats had longer convulsions as a result of stimulation of either primary or secondary sites. In this respect, we also observed that although the end of convulsions is easily determined in normal animals, in splitbrain animals this was not always the case. Instead of a definite termination of the clonus, split-brain animals may show a gradual decrease in the intensity of the seizure, so that several seconds after the termination of the clonus these animals show brief whole-body jerks every 5-8 seconds. In some cases, this continued for almost 2 minutes after the cessation of the clonus.

These results appear to indicate that some components of the overall kindling response are affected by split-brain surgery while others are not. Thus, it is possible that two independent systems subserve the kindling effect. The fact that convulsions are restricted to one side of the body in split-brain animals would argue against the hypothesis that the two sites share a common final seizure pathway (McIntyre and Goddard, 1973) and would suggest that normal bilateral convulsions are the result of the activation of two separate seizure pathways. In any case, the present data suggest that splitbrain surgery interferes markedly with inhibitory components of the kindling response, as indicated both by the longer duration of convulsions and faster initial kindling rates in these animals.

It might be tentatively proposed that two types of interhemispheric connections exist or become estab- 


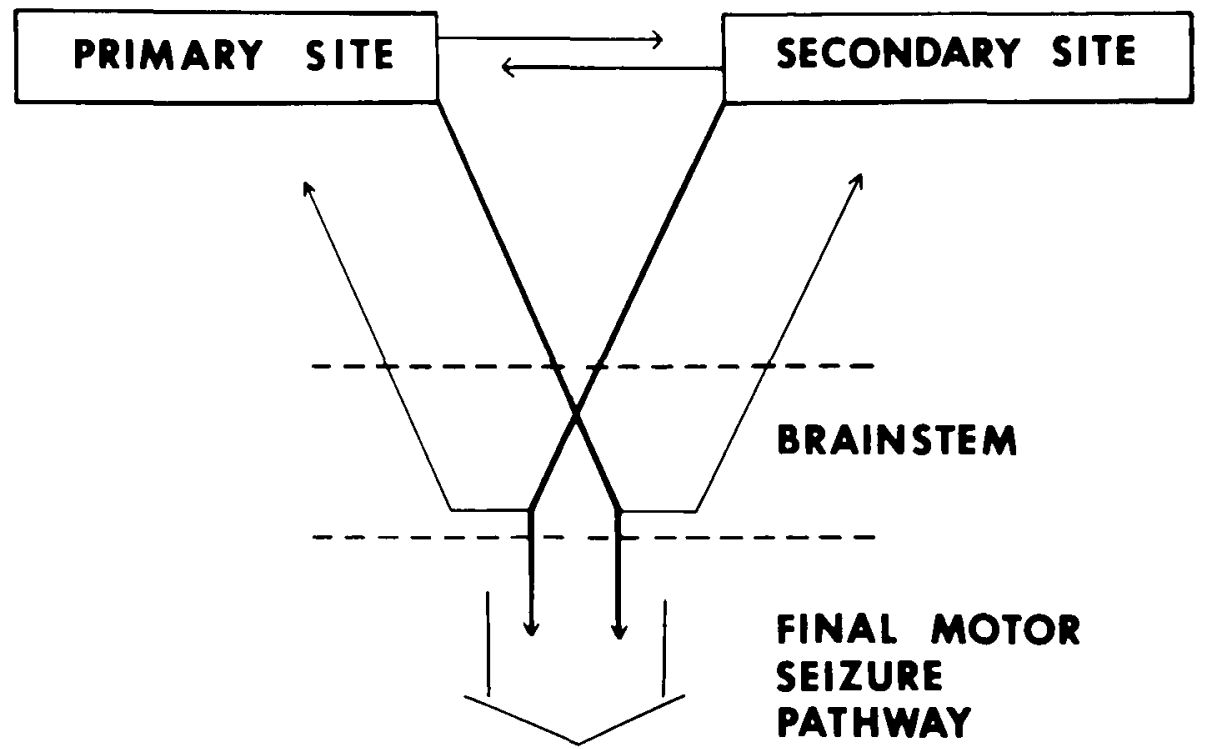

Figure 4-Diagrammatic representation of possible interhemispheric kindling pathways.

lished between primary and secondary sites, a short one via forebrain commissures and a long one via brainstem structures. If the additional supposition is made that each site develops its own motor seizure pathway, the model schematically presented in Figure 4 might represent an adequate concept of kindling pathways.

Here stimulation of the primary site would activate the secondary site through the short connections and simultaneously establish the contralateral component of the final seizure pathway. The indirect activation of the secondary site would, in turn, partially inhibit the primary site and establish the secondary, contralateral seizure pathway at the final brainstem level. In normal rats, such inhibitory effect from the secondary site on the primary would be eventually overridden by the imposition of current on the primary site, and bilateral convulsions would result from the simultaneous activation of the two components of the final seizure pathway. When stimulation is switched to the previously activated secondary site, fewer trials should be necessary to achieve CC's there. That stimulation of one amygdala induces changes in the electrical activity of the contralateral amygdala has been amply demon- strated in bilateral kindling studies (Racine, 1972).

In split-brain rats, the short connection between the two sites would be disrupted and, as a result, stimulation of each site should produce unilateral seizures The absence of negative feedback from the contralateral site would permit the achievement of the $\mathrm{CC}$ stage more rapidly, and conceivably also allow for longer convulsions. Stimulation of the contralateral site would be equivalent to initial kindling, for that site would not have been preactivated. This is in agreement with the observed absence of after-discharges in the secondary site during kindling of the primary site in splitbrain rats (McIntyre, 1975).

In terms of this model, oscillation would result from differences in the relative strengths of both long and short connections between the two sites. Although we stress the tentative and preliminary character of this hypothesis, we should note that it does lend itself to empirical verification. For example, the hypothesis would predict that temporary, reversible inactivation of one site (e.g., through cryogenic techniques or sub-cortical spreading depression) should have effects similar to those produced by split-brain surgery, that is, provide faster kindling rates, longer convulsions, and possibly also suppression of secondary oscillation. If proven correct, this model might provide a useful framework for an understanding of kindlingrelated phenomena.

\section{REFERENCES}

CULLEN, N., and GODDARD, G. V. (1975). Kindling in the hypothalamus and transfer to the ipsilateral amygdala. Behavioral Biology, 15, 119-131.

GiATO, J. (1974). The kindling effect. Physiological Psychology. 2. 45-50.

GAITO. J. (1976). An oscillation effect during sequential alternation of unilateral amygdaloid stimulation within the kindling paradigm. Physiological Psychology, 4, 303-306. (a).

GAITO. J. (1976). The effect of number of trials per day during sequential alternation of unilateral amygdaloid stimulation. Bulletin of the Psychonomic Society, 4, 403-404. (b).

GAITO, J. (1976). The effect of varying rest intervals following the development of oscillation during unilateral amygdaloid stimulation. Bulletin of the Psychonomic Society, 8, 457-458. (c).

GAITO. J. (1977). The effect of intensity during sequential alternation of unilateral amygdaloid stimulation. Bulletin of the Psychonomic Society, 9. 64-66.

GAITO, J., and NOBREGA. J. (1977). The oscillation effect during sequential alternation of amygdaloid stimulation with aged rats. Bulletin of the Psychonomic Society. 9. 151-154.

GAITO, J., GAITO, S. T., and NOBREGA. J. (1977). A factor analysis on data from 10 phases of sequential alternation. Physiological Psychology. 3, 300-310.

GODDARD. G. V., and DOUGLAS. R. M. (1975). Does the engram of kindling model the engram of normal long term memory? Canadian Journal of Neurological Sciences. 2. 385-394.

GODDARD. G. V.. McINTYRE. D. C., and LEECH, C. K. (1969). A permanent change in brain function resulting from daily electrical stimulation. Experimental Neurology. 25. 295-300.

HOEL. P. G. (1954). Introduction to mathematical statistics. New York: Wiley.

McCAUGHRAN. J. A.. CORCORAN, M. E., and WADA, J. A. Development of kindled amygdaloid seizures after section of the forebrain commissures in rats. Folia Psychiatrica et Neurologia Japonica, 30, 65-71.

MCINTYRE, D. C. (1975). Split-brain rat: transfer and interference of kindled amyg. dala convulsions. Canadian Journal of Neurological Sciences, 2, 429-437. 
McINTYRE, D. C., and GODDARD, G. V. (1973). Transfer, interference and spontaneous recovery of convulsions kindled from the rat amygdala. Electroencephalography and Clinical Neurophysiology, 35, 533-543.
RACINE, R. J. (1972). Modification of seizure activity by electrical stimulation: II. Motor seizure. Electroencephalography and Clinical Neurophysiology, 32, 281-294.

TRESS, K., and HERBERG, L. J. (1972). Permanent reduction in seizure threshold resulting from repeated electrical stimulation. Experimental Neurology, 37, 347-359.

WADA, J. A., and SATO, M. (1975). The generalized convulsive state induced by daily electrical stimulation of the amygdala in split brain cats. Epilepsia, 16, 417-430. 